\title{
DIVERSIDAD DE INVERTEBRADOS ACUÁTICOS DE LA BOCATOMA DE LA ATARJEA EN EL RÍO RÍMAC, LIMA PERÚ DURANTE 1999
}

\author{
Lorena Alvariño ${ }^{1,2}$ \\ José Iannacone $e^{3}$
}

\begin{abstract}
RESUMEN
El río Rímac es la principal fuente de agua potable para la ciudad de Lima, Perú, por lo que se evaluó la diversidad de invertebrados acuáticos en la bocatoma de la Atarjea, durante 1999. Se empleó una malla zooplanctónica de $45 \mu$, filtrándose 2 $\mathrm{L}$ de agua por muestra. Para la identificación y cuantificación de los invertebrados se empleó la cámara de Sedgewick-Rafter. El número total de organismos/L (org. $\mathrm{L}^{-1}$ ) fluctúo entre 0 (marzo) a 337,5 (noviembre). El promedio fue de 64,3 \pm 69,2. El grupo más abundante y frecuente fue rotífera con 11 especies. El 50\% de las muestras indicó una dominancia relativa por rotífera, el 36,3\% por nemátoda, 9,1\% para copépoda, $4,5 \%$ para protozoos e insectos, respectivamente. Los org $\cdot \mathrm{L}^{-}$ ${ }^{1}$ para rotífera fueron de $22,6 \pm 41,9$, para nemátoda de $25,4 \pm 31,7$ y para insecta de $3,1 \pm 5,3$. No se encontraron diferencias estacionales en el número de org $\mathrm{L}^{-1}$. Los mayores valores de equidad se encontraron en verano. Los índices de Jaccard y Sörensen mostraron una mayor similaridad entre invierno y primavera. La presencia de Ancylostoma duodenale Dubini, 1843, Chilomastix mesnili (Wenyon, 1910) Alexieieff, 1912 y Entamoeba coli (Gras, 1879) Casagrandi \& Barbagallo, 1895 indicaron contaminación fecal en el río Rímac.
\end{abstract}

Palabras claves: Copépoda, nemátoda, rotífera.

Laboratorio de Invertebrados. Facultad de Ciencias Biológicas. Universidad Ricardo Palma, Av. Benavides 5440, Santiago de Surco, Lima, Perú.

lalvarino@sedapal.com.pe

joseiannacone@gmail.com 


\section{SUMMARY}

Rimac river is the main source of potable water to Lima city, Peru, for this reason was evaluated the diversity of aquatic invertebrates in bocatoma of La Atarjea, during 1999. A zooplanktonic mesh of $45 \mu$, filtering $2 \mathrm{~L}$ of water by sample was employed. To identificate and quantificate invertebrates a Sedgewick-Rafter chamber was used. Total number of organisms $/ \mathrm{L}\left(\mathrm{org} \cdot \mathrm{L}^{-1}\right)$ fluctuated between 0 (March) to 337,5 (November). The mean was $64.3 \pm 69.2$. The group more abundant and frequent was rotifer with 11 species. $50 \%$ of the samples indicated a relative dominance of rotifers, $36.3 \%$ to nematod, $9.1 \%$ to copepod, $4.5 \%$ to protozoa and insects, respectively. Number of org $\mathrm{L}^{-1}$ for rotífer was $22.6 \pm 41.9$, for nematod was $25.4 \pm 31.7$ and for insect 3.1 \pm 5.3. Seasonal differences between number of org $\mathrm{L}^{-1}$ was not found. The highest values of eveness was found in summer. Jaccard and Sörensen indexes showed a higher similarity between winter and spring. Presence of Ancylostoma duodenale Dubini, 1843, Chilomastix mesnili (Wenyon, 1910) Alexieieff, 1912 and Entamoeba coli (Gras, 1879) Casagrandi \& Barbagallo, 1895 indicated fecal contamination in Rímac river.

Key words: copepod, nematode, rotifer.

\section{INTRODUCCIÓN}

Las comunidades zooplanctónicas son habitantes normales y constantes de casi todas las aguas superficiales naturales (Zambrano \& Burger, 1992; Fussman, 1996; Medina et al., 1999; Nielsen et al., 2000; Xie \& Yang, 2000). Los invertebrados componentes de la cadena trófica acuática cumplen un importante rol como descomponedores de la materia orgánica, así como depredadores y competidores (Medina et al., 1996; Finlay \& Esteban, 1998). Lima, la capital del Perú, depende principalmente para su abastecimiento de agua de los recursos del Río Rímac (La Touche, 1997). A pesar de que Lima, está localizada en el trópico, la ciudad tiene un clima templado con una temperatura media anual de $18^{\circ} \mathrm{C}$, debido a la corriente fría de Humboldt, la cual se desplaza por las costas de Sudamérica del antártico. La organización que es responsable para el abastecimiento del agua y la disposición del desagüe en esta ciudad es el Servicio de Agua Potable y Alcantarillado de Lima (SEDAPAL).

La comunidad de invertebrados en el río Rímac a la altura de la bocatoma de 
la Atarjea como punto de ingreso al Tratamiento de Agua Potable es importante, pues pudiera afectar el proceso y la calidad organoléptica del agua procesada (Wong \& Dixon, 1995; Paredes et al., 2005). La determinación cualitativa y cuantitativa de la comunidad de invertebrados nos sirven como indicadores biológicos de la calidad del agua (Cauchie et al., 2000); así como su rol como transportadores mecánicos de bacterias u otros patógenos por vía externa e interna (Angeler et al., 2000; Kim et al., 2000).

La composición y abundancia de las comunidades zooplanctónicas puede ser influenciada por númerosos factores físicos, químicos y biológicos (Bini et al., 2001; Bledzki \& Ellison, 2003; Rejas et al., 2005; Suarez-Morales et al., 2005). La temperatura, el tamaño de los cuerpos de agua, el estado trófico, el estado sucesional, la calidad y la disponibilidad de alimento, la competencia y la depredación pueden afectar tanto la composición como la densidad poblacional del zooplancton, y ser factores que actúen en simultáneo en los ambientes naturales (Rodríguez \& Matsumura-Tundisi, 2000; Sampaio et al., 2002; Arora \& Mehra, 2003; Nagorskaya, 2004; De Meester et al., 2005).

Los principales grupos componentes de las comunidades zooplanctónicas son los protozoos, rotíferos y crustá- ceos, particularmente copépodos y cladóceros. Sin embargo, el zooplancton dulceacuícola, especialmente en las regiones tropicales, puede contener una amplia diversidad de categorías taxonómicas (Santos-Wisniewski et al., 2002; Aoyagui et al., 2003; Suárez-Morales et al., 2004).

De esta forma, el objetivo de la presente investigación fue evaluar la diversidad de invertebrados acuáticos en el río Rímac en la bocatoma de la Atarjea-SEDAPAL durante 1999.

\section{MATERIAL Y MÉTODOS}

\section{Muestreo biológico}

Se emplearon recipientes de plástico de $120 \mathrm{~mL}$ de capacidad de boca ancha y con tapa de plástico hermética. El punto de muestreo fue en las ventanas de captación de la bocatoma de la Atarjea de la Cuenca baja del río Rímac, Lima, Perú. Se extrajo con una frecuencia aproximada quincenalmente un volumen de $2 \mathrm{~L}$, el cual fue filtrado a través de una malla de zooplancton cónica de $45 \mu$ de porosidad. El sedimento que quedó en la malla fue vertido en un frasco de plástico de $120 \mathrm{~mL}$. Posteriormente, se llevó la muestra al laboratorio y se fijó con formol azucarado al 10\%, con el fin de obtener una dilución al 50\%, quedando finalmente la muestra fijada al formol al 5\%. Se realizaron un total de 
24 muestreos de enero a diciembre de 1999. Todas las muestras se tomaron entre 09:00 a 11:00 h.

\begin{tabular}{cc}
\hline $\begin{array}{c}\text { Número de mues- } \\
\text { treo }\end{array}$ & Fecha de Muestreo \\
\hline 1 & 12 -enero \\
2 & 20 -enero \\
3 & 05 -febrero \\
4 & 15 -febrero \\
5 & 09 -marzo \\
6 & 16 -marzo \\
7 & 09 -abril \\
8 & 15 -abril \\
9 & 14 -mayo \\
10 & 18 -mayo \\
11 & 08 -junio \\
12 & 14 -junio \\
13 & 08 -julio \\
14 & 12 -julio \\
15 & 13 -agosto \\
16 & 22 -agosto \\
17 & 10 -septiembre \\
18 & 14 -septiembre \\
19 & 14 -octubre \\
20 & 20 -octubre \\
21 & 03 -noviembre \\
22 & 16 -noviembre \\
23 & 01 -diciembre \\
24 & 14 -diciembre \\
\hline
\end{tabular}

\section{Análisis cualitativo y cuantitativo}

Se realizó con la ayuda de un microscopio estereoscopio y con la cámara de Sedgewick-Rafter. Para la observación de los protozoarios se agregó unas gotas de lugol o se usó rojo neutro. Además se empleó el microscopio de contraste de fases para la identificación taxonómica de las especies.
Para la identificación de los especímenes se utilizaron los trabajos sobre copépodos de vida libre en el Perú (Del Río \& Valdivia, 1989). Para la identificación de los rotíferos se empleó a Koste (1972, 1978), a José de Paggi (1989) y a Segers (1995). Para los copépodos se usó a Holynska et al. (2003), a Mirabdullayev et al. (2003) y a Suarez-Morales et al. (2004, 2005). Para el resto de grupos de empleó literatura especializada para su identificación y la colaboración de profesionales especialistas.

Con el objetivo de verificar la constancia de cada taxón en las diferentes estaciones de muestreo, fue determinado el índice de constancia (Sampaio et al., 2002). Los taxones fueron considerados constantes (C) cuando fueron registrados en más del $50 \%$ de las muestras, comunes (c) cuando se presentaron entre un $10 \mathrm{y}$ $50 \%$, y finalmente raros (r) hasta en un $10 \%$ de las muestras. En adición se determinó la frecuencia de dominancia de los principales grupos de invertebrados (Iannacone \& Alvariño, 2007).

Cámara Sedgewick-Rafter: Se tomó una alícuota de la muestra con una pipeta de $1 \mathrm{~mL}$; si la muestra presentaba gran cantidad de materia orgánica, entonces se descargó a la cámara por una de las esquinas. La determinación cuantitativa sólo fue 
posible al enumerar en franjas o en campos separados, dependiendo de la densidad de la muestra.

Enumeración en franjas. Se usó la fórmula para determinar el número de organismos por $2 \mathrm{~L}$ o por el volumen en $L$ que se ha necesitado para concentrar la muestra en el campo.

$$
\begin{gathered}
\operatorname{org} \cdot \mathrm{L}^{-1}=\mathrm{C} \text { x } 1000 \mathrm{~mm}^{3} / \mathrm{L} \text { x P x A x } \\
\mathrm{N}
\end{gathered}
$$

Donde: $\mathrm{C}=$ Número de organismos contados. $\mathrm{L}=$ Longitud de cada tira (20 mm). P = Profundidad de cada tira $(1 \mathrm{~mm}) . \mathrm{A}=$ Ancho de cada tira. $\mathrm{N}$ $=$ Número de tiras contadas.

Enumeración en campos separados. Se usó la siguiente formula para la determinación de organismos invertebrados por $\mathrm{mL}$ o por el volumen en $\mathrm{L}$ que se ha necesitado para concentrar la muestra de campo.

$$
\operatorname{org} \cdot \mathrm{L}^{-1}=\mathrm{C} \times 1000 \mathrm{~mm}^{3} / \mathrm{A} \times \mathrm{P} \times \mathrm{N}
$$

Donde: $\mathrm{C}=$ Número de organismos contados. $\mathrm{A}=$ Área del campo del ocular de Whipple, 0,6084 $\mathrm{mm}$ u otra calibración. $\mathrm{P}=$ Profundidad del campo $1 \mathrm{~mm}$. $\mathrm{N}=$ Número de campos contados.

Finalmente los resultados se expresaron como organismos por $\mathrm{L}\left(\operatorname{org} \cdot \mathrm{L}^{-1}\right)$.

\section{Análisis estadístico}

Con el fin de evaluar la diversidad ecológica alfa $(\alpha)$ en las comunidades de cada una de las 24 evaluaciones y compararlas entre ellas, fueron utilizados los siguientes cinco índices: Shannon-wiever $\left(\mathrm{H}^{\prime}\right)$, Equidad de Pielou (J), Mc Intosh (M), Simpson (C) y Berger-Park (Margalef, 1991; Moreno, 2001).

Se emplearon tres métodos no paramétricos: Chao-2, Jacknife de primero orden y Jacknife de segundo orden para estimar el número máximo de riqueza de taxas de invertebrados total a partir del esfuerzo de muestreo realizado y de la acumulación de taxas nuevos que van apareciendo en las muestras (Moreno, 2001).

Se hicieron comparaciones cualitativas entre las 4 estaciones del año basadas en la presencia y ausencia de especies, de acuerdo con los índices de similaridad de Sörensen y de Jaccard (Moreno, 2001).

Se utilizó el Análisis de Varianza (ANDEVA, modelo aditivo lineal de una vía), empleando el estadístico de Fisher $(\mathrm{F})$, en un diseño experimental completamente randomizado (DCR) con el fin de comparar el promedio del número de org $\cdot \mathrm{L}^{-1}$, especies $\cdot \mathrm{L}^{-1}$, Índice de Simpson, Índice de diversidad Mc Intosh, Índice de Berger-Parker, principales grupos taxonómicos y especies más frecuentes entre las cuatro estaciones (enero-marzo; abril-junio; julio- 
septiembre; octubre-diciembre). Además, la correlación de Pearson (r) se utilizó para determinar la relación lineal entre los grupos taxonómicos más dominantes. El nivel de significancia empleado fue $\alpha=0,05$ (Zar, 1996).

\section{RESULTADOS}

21 taxas pertenecientes a la fauna de invertebrados en el río Rímac a la altura de la bocatoma, La Atarjea, Lima, Perú fueron encontrados durante 1999 (Tabla 1). De los taxas encontrados 11 fueron rotíferos, 3 fueron protozoarios de vida libre, 2 nematodos, 2 insectos, 1 copépodo, 1 ostracoda, y 1 anélida. En adición se encontraron dos protozoarios parásitos no patógenos $\mathrm{y}$ huevos del nemátodo Ancylostoma duodenale; así como Chironomus calligraphus en forma de huevo, larva y pupa (Tabla 1). Los taxas Rhabdolaimus terrestris (70,8\%; $\mathrm{n}=17), C$. calligraphus $(45,8 \% ; \quad \mathrm{n}=11)$, Brachionus calyciflorus $(33,3 \% ; \mathrm{n}=8), B$. dimidiatus $(33,3 \% ; \mathrm{n}=8)$ y Epiphanes macrourus $(33,3 \% ; \mathrm{n}=8)$ fueron los taxa más frecuentes, respectivamente (Tabla 1).

Con relación a la frecuencia de dominancia se encontró la siguiente secuencia en orden decreciente: rotífera, nemátoda, copépoda, protozoa e insecta (Tabla 2). En adición, nemátoda y rotífera presentaron por igual una sola frecuencia de dominancia compartida (Tabla 2).

Con relación la estacionalidad, se encontró un mayor número de especies en julio-septiembre (invierno), y un mayor número de especimenes entre octubrediciembre (primavera) de 1999 (Tabla 3). Con relación a la diversidad alfa, el índice de Shannon-Wiever presentó la siguiente secuencia en orden descendente: primavera $\left(\mathrm{H}^{\prime}=0,86\right)$, verano $\left(\mathrm{H}^{\prime}=0,79\right)$, otoño $\left(\mathrm{H}^{\prime}=0,79\right)$ e invierno $\left(\mathrm{H}^{\prime}=0,77\right)$. En adición, el índice de equidad mostró la siguiente secuencia en orden descendente: verano $(\mathrm{J}=0,88)$, otoñó $(\mathrm{J}=0,76)$, primavera $(\mathrm{J}=0,75)$ e invierno $(\mathrm{J}=0,74)$. Los índices de McIntosh y de Simpson mostraron la misma secuencia en orden decreciente: invierno, primavera, otoño y verano. La frecuencia de valores de los índices de McIntosh y de Simpson resultaron relativamente altos para los invertebrados acuáticos de la bocatoma del río Rímac durante 1999 (Figs. 1 y 2). El índice de Berger-Parker presentó la siguiente secuencia en orden descendente: primavera, otoño, invierno y verano (Tabla 3). Los rotífera, los nemátoda, $R$. terrestris y B. calyciflorus presentaron las mayores cantidades de especimenes en primavera, aunque con niveles estadísticamente no significativos. En cambio, insecta y E. macrourus presentaron el mayor número de especimenes en otoño e invierno, respectivamente (Tabla 3). 


$$
\text { III }
$$


Tabla $N^{\circ}$ 2: Frecuencia de dominancia de los principales grupos de invertebrados

\begin{tabular}{ccc}
\hline Taxones & $\begin{array}{c}\text { Frecuencia de } \\
\text { Dominancia }\end{array}$ & $\begin{array}{c}\text { Frecuencia de dominancia } \\
\text { compartida por dos grupos }\end{array}$ \\
\hline Protozoa & 1 & 0 \\
Nemátoda & 7 & 1 \\
Rotífera & 10 & 1 \\
Annelida & 0 & 0 \\
Copépoda & 2 & 0 \\
Ostrácoda & 0 & 0 \\
Insecta & 1 & 0 \\
\hline
\end{tabular}

Tabla No 3: Fluctuación estacional del número de especies, especímenes, índices de diversidad alfa, principales grupos taxonómicos y especies más frecuentes en la bocatoma del río Rímac, Lima, Perú.

\begin{tabular}{|c|c|c|c|c|c|c|c|c|c|c|c|}
\hline Semestres & 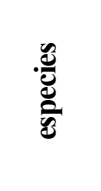 & & 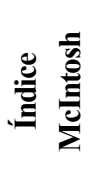 & 异总 & 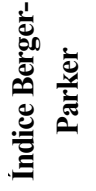 & 弟 & 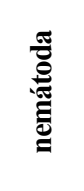 & 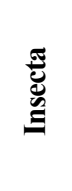 & $\approx$ ¿ & 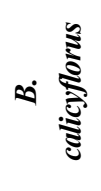 & 小 \\
\hline enero-marzo & $2,5^{\mathrm{a}}$ & $18,5^{\mathrm{a}}$ & $0,29^{\mathrm{a}}$ & $0,36^{\mathrm{a}}$ & $0,34^{\mathrm{a}}$ & $11,0^{\mathrm{a}}$ & $4,5^{\mathrm{a}}$ & $2,3^{\mathrm{a}}$ & $4,5^{\mathrm{a}}$ & $0,6^{\mathrm{a}}$ & $1,8^{\mathrm{a}}$ \\
\hline abril-junio & $3,5^{\mathrm{a}}$ & $77,5^{\mathrm{a}}$ & $0,33^{\mathrm{a}}$ & $0,54^{\mathrm{a}}$ & $0,58^{\mathrm{a}}$ & $14,8^{\mathrm{a}}$ & $31,1^{\mathrm{a}}$ & $7,2^{\mathrm{a}}$ & $15,5^{\mathrm{a}}$ & $1,5^{\mathrm{a}}$ & $0^{\mathrm{a}}$ \\
\hline julio-septiembre & $5,1^{\mathrm{a}}$ & $61^{\mathrm{a}}$ & $0,45^{\mathrm{a}}$ & $0,58^{\mathrm{a}}$ & $0,54^{\mathrm{a}}$ & $37,2^{\mathrm{a}}$ & $17,5^{\mathrm{a}}$ & $0,9^{\mathrm{a}}$ & $30,5^{\mathrm{a}}$ & $30,5^{\mathrm{a}}$ & $11,5^{\mathrm{b}}$ \\
\hline octubre-diciembre & $4,3^{\mathrm{a}}$ & $100,1^{\mathrm{a}}$ & $0,42^{\mathrm{a}}$ & $0,57^{\mathrm{a}}$ & $0,59^{\mathrm{a}}$ & $38,7^{\mathrm{a}}$ & $37,4^{\mathrm{a}}$ & $2,1^{\mathrm{a}}$ & $38,9^{\mathrm{a}}$ & $38,9^{\mathrm{a}}$ & $4,4^{\mathrm{a}}$ \\
\hline DF & 64,3 & 3,8 & 0,38 & 0,52 & 0,51 & 22,6 & 25,4 & 3,1 & 22,3 & 8,2 & 4,4 \\
\hline P IOIIECAO DE & $\pm 69,2$ & $\pm 2,0$ & $\pm 0,22$ & $\pm 0,27$ & $\pm 0,17$ & $\pm 41,9$ & $\pm 31,7$ & $\pm 5,3$ & $\pm 42,0$ & $\pm 19,6$ & $\pm 7,5$ \\
\hline $\mathrm{F}$ & 2,19 & 1,61 & 0,53 & 0,78 & 2,45 & 1,31 & 0,70 & 1,79 & 0,77 & 1,16 & 3,7 \\
\hline Probabilidad & 0,12 & 0,21 & 0,66 & 0,52 & 0,09 & 0,29 & 0,56 & 0,18 & 0,52 & 0,35 & 0,02 \\
\hline
\end{tabular}

Enero-marzo $=$ verano. Abril-junio $=$ otoño. Julio-septiembre $=$ invierno. Octubre-diciembre $=$ primavera. $\mathrm{F}=$ Prueba de Fisher. Letras minúsculas iguales en una misma columna indican que los promedios son estadísticamente iguales. $\mathrm{DE}=$ Desviación Estándar 


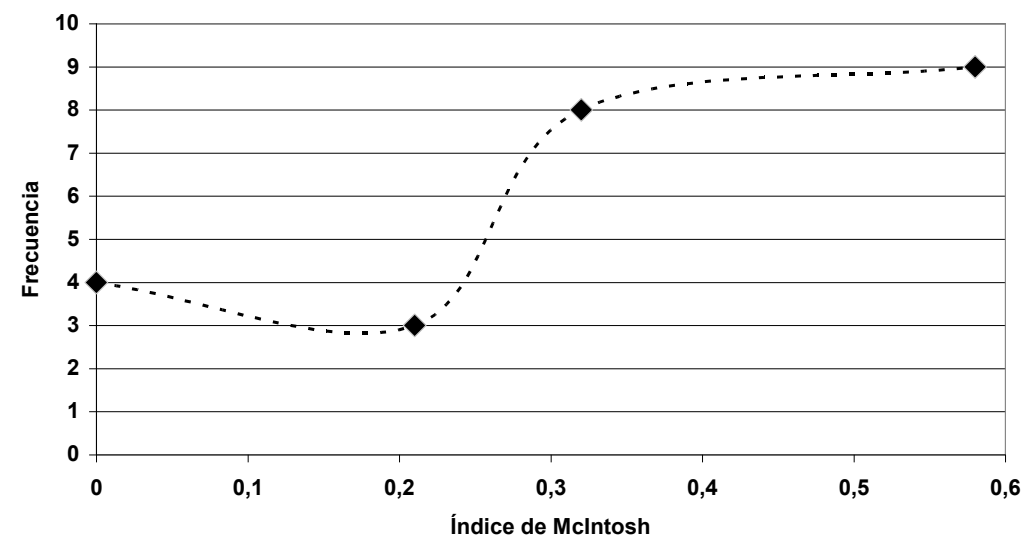

Fig. 1. Frecuencia de valores del Î́ndice de McIntosh de invertebrados acuáticos de la Bocatoma del río Rímac durante 1999

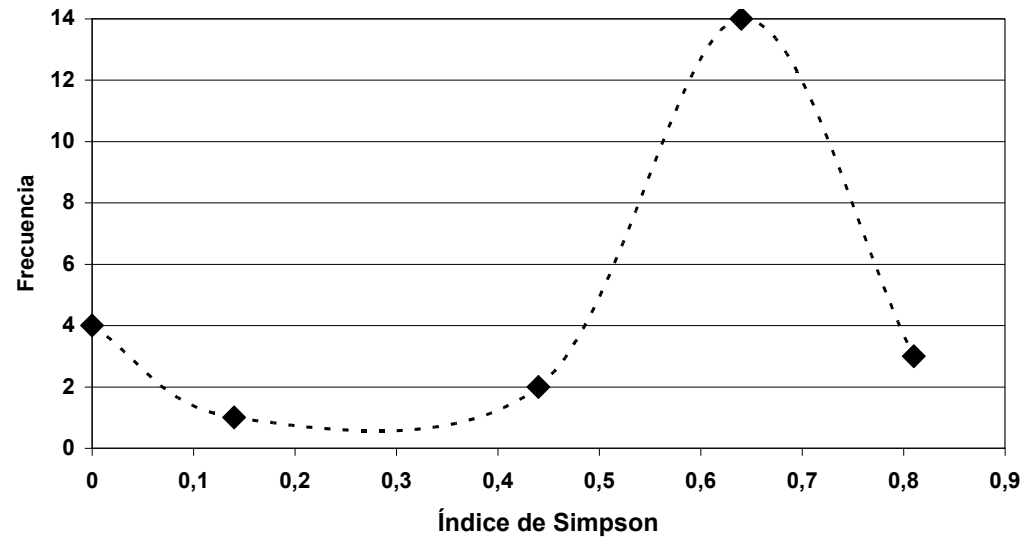

Fig. 2. Frecuencia de valores del Índice de Simpson de invertebrados acuáticos de la Bocatoma del río Rímac durante 1999

Se empleó los estimadores Chao-2, Jacknife -1 y Jacknife -2 para determinar el número de especies que podrían existir en la bocatoma de la Atarjea durante 1999, los cuales serían 29, 28 y 31 especies, respectivamente.

La Tabla 4, nos indica según los índices de similaridad de Sörensen y de Jaccard que los mayores valores se observaron entre invierno y primavera, así como entre verano y primavera, y entre otoño e invierno para ambos índices.

Se encontró relación lineal positiva entre rotífera y nemátoda en todas las muestras analizadas durante 1999 $(\mathrm{r}=0,46 ; \mathrm{P}=0,02 ; \mathrm{n}=24)$. No se encontró relación lineal entre rotífera e insecta; $\mathrm{y}$ tampoco entre nemátoda e insecta $(\mathrm{P}>$ $0,05)$. 
Tabla $N^{\circ}$ 4: Matriz de similaridad entre estaciones de 1999 para la fauna de invertebrados de la bocatoma, La Atarjea, Lima, Perú empleando los índices cualitativos de Sörensen y de Jaccard.

\begin{tabular}{cccccc} 
& \multicolumn{5}{c}{ Índice de Sörensen } \\
\cline { 2 - 6 } & & Verano & Otoño & Invierno & Primavera \\
Índice & Verano & - & 42,1 & 31,5 & 63,6 \\
de & Otoño & 26,6 & - & 63,5 & 40 \\
Jaccard & Invierno & 18,7 & 46,6 & - & 72 \\
& Primavera & 46,6 & 25 & 56,2 & - \\
\hline
\end{tabular}

enero a marzo $=$ verano $;$ abril a junio $=$ otoño $;$ julio a septiembre $=$ invierno $;$ octubre a diciembre $=$ primavera.

\section{DISCUSIÓN}

La contaminación del río Rímac, única fuente de abastecimiento de agua Potable de la ciudad de Lima, tiene su origen en diversas fuentes: industriales, domésticas, agrícolas, etc. que contienen sales de metales pesados como el plomo, cadmio, arsénico y cobre (Iannacone \& Alvariño, 1996; Iannacone et al., 2005), condiciones que influyen en la comunidad biológica existente en la Bocatoma La Atarjea, en especial en los invertebrados de vida libre. La presencia de $A$. duodenale, Chilomastix mesnili y Entamoeba coli indicaron contaminación fecal en este cuerpo de agua (Tabla 1) (Iannacone, 2002).

Los principales grupos que componen las comunidades zooplanctónicas son los protistas, rotíferos y crustáceos, particularmente copépodos y cladóceros (Sampaio et al. 2002; Iannacone
\& Alvariño, 2007). Los rotíferos tienden a ser los organismos zooplanctónicos numéricamente más importantes en el río, seguido de los nauplios, bosmínidos y copépodos ciclopoideos. Los valores más altos de densidad de los rotíferos en estos cuerpos de agua se han registrado durante los periodos de bajos niveles del agua (Aoyagui \& Bonecker, 2004). Esta relación inversa, podría ser el resultado de un efecto de concentración del organismo, también por la ocurrencia de condiciones favorables a su desarrollo, como la disminución de la velocidad de la corriente del agua y la concentración de sólidos suspendidos. Estos factores físicos pueden favorecer el desarrollo del fitoplancton, uno de los principales recursos para los rotíferos (Aoyagui \& Bonecker, 2004). Los rotíferos fueron el grupo con mayor riqueza taxonómica y abundancia durante todo el periodo de 
muestreo (Tablas 1 y 2). Resultados similares han sido obtenidos por Sampaio et al. (2002) y Iannacone \& Alvariño (2007). Sin embargo, los rotíferos no mostraron patrones estacionales muy marcados, a excepción de E. macrourus (Tabla 3). Este patrón de mayor riqueza taxonómica y abundancia es el común en ambientes dulceacuícolas tropicales, sean estos lagos, lagunas, reservorios, ríos o arroyos. Esto puede ser atribuido a que estos organismos son estrategas $r$, oportunistas, de tamaño pequeño, ciclo de vida corto y amplia tolerancia a una variedad de factores ambientales (Iannacone \& Alvariño, 2007). En adición, el amplio espectro de partículas alimenticias explotadas por este grupo, el cual tiene la habilidad de consumir bacterias, algas y detritus de diferentes tamaños, le permite diferentes dietas para muchas especies simultáneamente presentes en los cuerpos de agua (Arora \& Mehra 2003).

Rhabdolaimus terrestris, es un nematodo bacterívoro muy abundante en los ambientes dulceacuícolas y terrestres (Iannacone \& Alvariño, 2007). En el presente trabajo fue la única especie considerada común (Tablas $1 \mathrm{y}$ 2).

La diversidad beta de similaridad entre las cuatro estaciones indicó valores no muy cercanos entre sí, fluctuando entre el $0,1 \%$ y el $40,5 \%$ entre los dos índices cualitativos (Tabla 4). Los tres estimadores empleados Chao-2, Jacknife -1 y Jacknife -2 indican la necesidad de continuar censando y analizando la diversidad de invertebrados de la bocatoma de la Atarjea, Lima, Perú para conclusiones más definitivas (Paredes et al., 2007).

\section{CONCLUSIONES}

Al evaluar la diversidad de invertebrados acuáticos en la bocatoma de la Atarjea, Lima, Perú durante 1999 se encontró lo siguiente:

- El número total de organismos/L (org $\cdot \mathrm{L}^{-1}$ ) fluctúo entre 0 (marzo) a 337,5 (noviembre). El promedio fue de $64,3 \pm 69,2$. El grupo más abundante y frecuente fue rotífera con 11 especies. El 50\% de las muestras indicó una dominancia relativa por rotífera, el $36,3 \%$ por nemátoda, 9,1\% para copépoda, 4,5\% para protozoos e insectos, respectivamente.

- Los org $\mathrm{L}^{-1}$ para rotífera fueron de 22,6 4 41,9, para nemátoda de $25,4 \pm 31,7$ y para insecta de $3,1 \pm$ 5,3 .

- No se encontraron diferencias estacionales durante 1999 en el número de org $\cdot \mathrm{L}^{-1}$. 
- Los mayores valores de equidad se encontraron en verano.

- Los índices de Jaccard y Sörensen mostraron una mayor similaridad entre invierno y primavera.

- La presencia de Ancylostoma duodenale, Chilomastix mesnili y Entamoeba coli indicaron contaminación fecal en el río Rímac.

\section{LITERATURA CITADA}

ANGELER, D.G.; ALVAREZCOBELAS, M.; ROJO, C. \& SÁNCHEZ-CARRILLO, S. 2000. The significance of water inputs to plankton biomass and trophic relationships in a semi-arid freshwater wetland (Central Spain). Journal of Plankton Research. 22: 20752093.

AOYAGUI, A.S.M.; BONECKER, C.C.; LANSAC-TÔHA, F.A. \& VELHO, L.F.M. 2003. Structure and dynamics of rotifers in Corumbá reservoir, state of GóiasBrazil. Acta Scientiarum. 25: 3139.

AOYAGUI, A.S.M. \& BONECKER, C.C. 2004. The art status of rotifer studies in natural environments of South America: floodplains. Acta Scientiarum. 26: 385-406

ARORA, J. \& MEHRA, N.K. 2003. Species diversity of planktonic and epiphytic rotifers in the backwaters of the Delhi segment of the
Yamuna Rivers, with remarks on new records from India. Zoological studies. 42: 239-247.

BINI, L.M.; BONECKER, C.C. \& LANSAC-TÔHA, F.A. 2001. Vertical distribution of rotifers on the upper Paraná River floodplain: the rol of thermal stratification and chlorophyll-a. Studies on Neotropical Fauna and Environment. 36: 241-246.

BLEDZKI, L.A. \& ELLISON, A.M. 2003. Diversity of rotifers from northeastern U.S.A. bogs with new species records for North America and New England. Hidrobiología. 497: 53-62.

CAUCHIE, H.M.; HOFFMAN, L. \& THOMÉ, J.P. 2000. Metazooplankton dynamics and secondary aerated waste stabilization pond. Journal of Plankton Research. 22: 2263-2287.

DEL RÍO, M. \& VALDIVIA, R. 1989. Copépodos dulceacuícolas de vida libre en el Perú. Familia Cyclopidae. Orden Cyclopoida. Boletín de Lima. 63: 71-80.

FINLAY, B.J. \& ESTEBAN, G.F. 1998. Freshwater protozoa: biodiversity and ecological function. Biodiversity \& Conservation. 7: 1163-1186.

FUSSMANN, G. 1996. The importance of crustacean zooplankton in structuring rotifer and phytoplankton communities: 
an enclosure study. Journal of Plankton Research. 18: 18971915.

HOLYNSKA, M.; REID, J.W. \& UEDA, H. 2003. Genus Mesocyclops Sars, 1914. Copepoda: Cyclopoida genera Mesocyclops and Thermocyclops (ed. By $\mathrm{H}$. Ueda and J.W. Reid), pp. 12-213. Guides to the Identification of the Microinvertebrates of the Continental Waters of the World. Backhuys Publishers, Amsterdam.

IANNACONE, J. 2002. Remoción de formas parasitarias intestinales en una laguna facultativa de estabilización de Lima, Perú. Revista brasileira de Zoología. 19: 10331041.

IANNACONE, J. \& ALVARIÑO, L. 1986. La lombriz roja Chironomus calligraphus y la pulga del agua dulce Moina macrocopa como herramienta ecotoxicológica para la evaluación del lindano y clorpirifos. Hipótesis. 6:59-64.

IANNACONE, J. \& ALVARIÑO, L. 2007. Diversidad y abundancia de comunidades zooplanctónicas litorales del humedal Pantanos de Villa, Lima, Perú. Gayana. 71: 49-65.

IANNACONE, J.; ARRASCUE, A. \& CÁRDENAS, M. 2005. Evaluación de la ecotoxicidad de sedimentos del río Aruri y del Lago Junín (Perú) usando
Escherichia coli y Chironomus calligraphus. Revista Brasileira de Toxicología. 18: 27-41.

JOSÉ DE PAGGI, S. 1989. Rotíferos de lagunas provincias del noroeste Argentino. Revista de Hidrobiologia Tropical. 22: 223-238.

KIM, H.W.; HWANG, S.J. \& JOO, G.J. 2000. Zooplankton grazing on bacteria and phytoplankton in a regulated large river (Nakdong River, Korea). Journal of Plankton Research. 22: 1559-1577.

KOSTE, W. 1972. Rotatorien aus Gewassen Amazoniens. Amazoniana. 3: 258-505.

KOSTE, W. 1978. Rotatoria. Die Rädertiere Mittleeuropas. Vol. 2. Stuttgart: Gebrüder Bomtraeger. Berlin. 673 p.

LA TOUCHE, M.A. 1997. The Water resources of Lima, Peru. Water and Environment Journal. 11: 437-439.

MARGALEF, R. 1991. Teoría de los sistemas ecológicos. Publicacions de la Universitat de Barcelona, Barcelona, España. 290 p.

MEDINA, S. J.M., VILLAR, A. M.; SÁNCHEZ C. P.; CRUZ, P. L. \& CARRILLO, P. 1999. Structure changes in a planktonic food web: biotic and abiotic controls. Journal of Limnology. 58: 213-222.

MEESTER, L.D. ; DECLERCK, S.; STOKS, R., LOUETTE, G.; VAN DE MEUTTER, F. ; DE BIE, T.; 
MICHELS, E. \& BRENDONCK, L. 2005. Ponds and pools as models systems in conservation biology, ecology and evolutionary biology. Aquatic Conservation: Marine and freshwater ecosystems. 15: 715-725.

MIRABDULLAYEV, I.M.; REID, J.W. \& UEDA, H. 2003. Genus Thermocyclops Kiefer, 1927. Copepoda: Cyclopoida genera Mesocyclops and Thermocyclops. En: Guides to the Identification of the Microinvertebrates of the Continental Waters of the World. (Eds. Ueda, H. \& Reid, J.W.), pp. 214302. Backhuys Publishers, Amsterdam.

MORENO, C.E. 2001. Métodos para medir la biodiversidad. Manuales y Tesis SEA. Sociedad Entomológica Aragonesa Ed. Madrid, España. 80 pp.

NAGORSKAYA， L. 2004. Living freshwater ostracoda (Crustacea) of a floodplain relict oak forest (Polesye, Belarus). Revista Española de Micropaleontología. 36: 135145.

NIELSEN, D.L.; SMITH, F.J.; HILLMAN, T.J. \& SHIEL, R.J. 2000. Impact of water regime and fish predation on zooplankton resting egg production and emergence. Journal of Plankton Research. 22: 433-446.
PAREDES, C.; IANNACONE, J. \& ALVARIÑO, L. 2005. Uso de macroinvertebrados bentónicos como bioindicadores de la calidad de agua en el río Rímac, Lima-Callao, Perú. Revista Colombiana de Entomología. 31: 219-225.

PAREDES, C.; IANNACONE, J. \& ALVARIÑO, L. 2007. Biodiversidad de invertebrados de los humedales de Puerto Viejo, Lima, Perú. Neotropical Helminthology. 1: 21-30.

REJAS, D.; DECLERCK, S.; AUWERKERKEN, J.; TAK, P. \& DE MEESTER, L. 2005. Plankton dynamics in a tropical floodplain lake: fish, nutrients, and the relative importance of bottom-up to top-down control. Freshwater Biology. 50: 52-69.

RODRIGUEZ, M.P. \& MATSUMURA-TUNDISI, T. 2000. Variation of density, species composition and dominance of rotifers at a shallow tropical reservoir (Broa reservoir, SP, Brazil) in a short scale time. Revista Brasileira de Biología. 60: 1-9.

SAMPAIO, E.V.; ROCHA, O.; MATSUMURA-TUNDISI, T. \& TUNDISI, J.G. 2002. Composition and abundance of zooplankton in the limnetic zone of seven reservoirs of the Paranapema River, Brazil. Brazilian Journal of Biology. 62: 525-545. 
SANTOS-WISNIEWSKI, M.J.; ROCHA, O.; GÜNTZEL, A.M. \& MATSUMURA-TUNDISI, T. 2002. Cladocera Chydoridae of high altitude water bodies (Serra da Mantiqueira), in Brazil. Brazilian Journal of Biology. 62: 681-687.

SEGERS, H. 1995. Rotifera 2: The Lecanidae (Monogononta). En: Guides to the identification of the microinvertebrates of the continental waters of the world 6. (Eds. H.J. Dumont, T. Nogrady). The Hagues: SPB Academic Publishing. The Netherlands. 226 pp.

SUAREZ-MORALES, E.; REID, J.W. ; FIERS, F. \& ILIFFE, T.M. 2004. Historical biogeography and distribution of the freshwater cyclopine copepods (Copepoda, Cyclopopida, Cyclopinae) of the Yucatan Peninsula, Mexico. Journal of Biogeo-graphy. 31: 10511063.

SUAREZ-MORALES, E.; REID, J.W. \& ELÍAS-GUTIERREZ, M. 2005.
Diversity and distributional patterns of Neotropical freshwater copepods (Calanoida: Diaptomidae). International Review of Hydrobiology. 90: 71-83.

WONG, P.T.S. \& D.G. DIXON. 1995. Bioassessment of water quality. Environmental Toxicology and $\mathrm{Wa}$ ter Quality. 10:9-17.

XIE, P. \& Y. YANG. 2000. Long-term changes of copepoda community (1957-1996) in a subtropical Chinese lake stocked densely with planktivorous filter-feeding silver and bighead carp. Journal of Plankton Research. 22: 1757-1778.

ZAMBRANO, F. \& BURGER, L. 1992. Notas comparativas sobre la diversidad zooplanctónica de diez lagunas altoandinas en Huánuco, Perú. Boletín de Lima. 14(84): 89-95.

ZAR, J. H. 1996. Bioestatistical anal$y$ sis. $3^{\text {th }}$ Ed. Prentice-Hall. Inc. Upper Saddle River, New Jersey. $662 \mathrm{p}$. 